\title{
THE EXACT RENORMALIZATION GROUP IN ASTROPHYSICS
}

\author{
JOSÉ GAITE \\ Centro de Astrobiología, CSIC-INTA, \\ Carretera Ajalvir, km 4, 28850 Torrejón de Ardoz, Madrid, Spain
}

\begin{abstract}
The coarse-graining operation in hydrodynamics is equivalent to a change of scale which can be formalized as a renormalization group transformation. In particular, its application to the probability distribution of a self-gravitating fluid yields an "exact renormalization group equation" of Fokker-Planck type. Since the time evolution of that distribution can also be described by a Fokker-Planck equation, we propose a connection between both equations, that is, a connection between scale and time evolution. We finally remark on the essentially non-perturbative nature of astrophysical problems, which suggests that the exact renormalization group is the adequate tool for them.
\end{abstract}

\section{Dynamical equations and fluctuations}

\subsection{Astrophysical hydrodynamics}

The usual hydrodynamic equations are the continuity equation, the Euler (or Navier-Stokes) equation describing momentum conservation, and the energy equation. To account for the gravitational field, we must add its corresponding equations, namely, the usual equations for its divergence and curl. In cosmology, we must also account for the global expansion, which, in the Newtonian formulation, introduces the scale factor $a(t)$ and the Hubble constant $H(t) .0$

Then we have five equations altogether to describe the evolution of the fields, but we assume that the solution of the energy equation is a polytropic equation of state $P=\kappa \varrho^{\gamma}$. So we have four equations:

$$
\begin{array}{r}
\frac{\partial \varrho}{\partial t}+3 H \varrho+\frac{1}{a} \nabla \cdot(\varrho \boldsymbol{v})=0, \\
\frac{\partial \boldsymbol{v}}{\partial t}+H \boldsymbol{v}+\frac{1}{a} \boldsymbol{v} \cdot \nabla \boldsymbol{v}=\boldsymbol{g}-\frac{1}{a} \frac{\nabla P}{\varrho}, \\
\nabla \cdot \boldsymbol{g}=-4 \pi G a\left(\varrho-\varrho_{b}\right), \quad \nabla \times \boldsymbol{g}=\mathbf{0}
\end{array}
$$

$\varrho_{b}$ is the background (average) density.

Of course, the two equations for the gravitational field can be reduced to a single equation for the gravitational potential. Then we have two scalar equations, that is, the continuity and the Poisson equation, and one vector equation, namely, the Euler velocity equation, for two scalar unknowns (the density and the gravitational potential) and one vector unknown (the velocity). $H, \varrho_{b}$ and $a$ are known functions of time in a definite cosmological model. For astrophysical situations in which the cosmological expansion is irrelevant, we may put $a=1$ and $\varrho_{b}=0, H=0$.

\subsection{Coarse graining}

The hydrodynamic equations are macroscopic equations, which can be assumed to follow from microscopic Newtonian mechanics of particles through an averaging 
process called coarse graining. The average density $\varrho_{L}(\boldsymbol{r})$ of an element of volume, say a box of side $L$, centered on the point $\boldsymbol{r}$ is the total mass of the particles inside divided by the volume $L^{3}$. One defines similarly the velocity field $\boldsymbol{v}_{L}(\boldsymbol{r})$, etc.

We must regard that the coarse graining operation does not completely eliminate the irregular nature of the microscopic variables but leaves a fluctuating component, which should vanish as $L$ grows. In general, the use of hydrodynamics implies a thermodynamic treatment of the element of volume, to which one can add the usual thermodynamic fluctuations. However, since the gravitational force is long ranged, the separation of scales on which the theory of thermodynamic fluctuations is based is useless here. Therefore, the hydrodynamic equations must be supplemented with the fluctuations, as usual in hydrodynamics, but with fluctuations that are of a particular nature..$^{a}$ As one scales $L$ up, the coarse-grained fields change, as well as the fluctuations. This is the essence of the renormalization group.

Besides stochastic fluctuations, which appear as noise terms in the hydrodynamic equations, one can also consider a probability distribution of initial fields, as usually done in cosmology. The fluctuations are determined by the correlation functions of the noise or the initial conditions.

\subsection{Dynamical evolution}

To solve a set of stochastic partial differential equations (SPDE), one must solve before the partial differential equations as if the noise were just an external source, and then express the correlation functions of the dynamical fields $\varrho$ and $\boldsymbol{v}$ in terms of the correlation functions of the noise (or initial conditions). Of course, the solution is of stochastic nature and can also be expressed in terms of a probability distribution $\mathcal{P}[\varrho, v, t]$ : the correlation functions are given by the corresponding functional integrals. The SPDE are therefore equivalent to an evolution equation for the probability distribution $\mathcal{P}$. We shall show below how to solve the hydrodynamic SPDE and the associated equation for $\mathcal{P}$ corresponding to a simplified version of the total set of equations.

\section{The exact renormalization group equation}

Apart from their time evolution, the coarse-grained variables change (we may also say "evolve") with the coarse-graining length $L$. ¿From a field-theory point of view, a smoothing of the "bare" fields $\varrho$ and $\boldsymbol{v}$ is necessary to regularize short-distance singularities in functional integrals. It is convenient in our context to define a general coarse graining procedure with the help of a window function, that is, a function that quickly vanishes outside a neighborhood of the origin of size $L$; typical examples are the sharp-cutoff ("top-hat") window and the Gaussian window. Thus, we define, for example, a smoothed density field as the convolution

$$
\varrho_{L}(\boldsymbol{r})=\int W_{L}(\boldsymbol{r}-\boldsymbol{x}) \varrho(\boldsymbol{x}) .
$$

${ }^{a} \mathrm{~A}$ recent fiscussion of hydrodynamic equations in the presence of gravitation is given by A. Domínguez.2 
As $L$ runs, the coarse-grained variables change and so does the probability distribution $\mathcal{P}$ (for simplicity, here we disregard its time dependence, as well as the variable $\boldsymbol{v}$, and write $\left.\mathcal{P}_{L}[\varrho]\right)$ :

$$
\frac{\partial}{\partial L} \mathcal{P}_{L}[\varrho]=\frac{d \ln \tilde{W}_{L}}{d L} \mathcal{P}_{L}[\varrho]-\frac{d \ln \tilde{W}_{L}^{2}}{d L} \frac{\delta}{\delta \varrho}\left(\varrho \mathcal{P}_{L}[\varrho]\right)-\frac{1}{2} \frac{d \tilde{W}_{L}^{2}}{d L} \frac{\delta^{2}}{\delta \varrho^{2}} \mathcal{P}_{L}[\varrho]
$$

where $\tilde{W}_{L}$ is the Fourier transform of the window function. This differential equation, describing evolution with the scale $L$, is the exact renormalization group equation for $\mathcal{P}_{L}$. Its proof is exposed in the appendix. A useful remark is that it is a sort of Fokker-Planck equation for $L$-evolution.

The exact renormalization group equation relies on Wilson non-perturbative philosophy of the renormalization group and, in fact, was proposed by Wilson himself 3 It has been amply used in high-energy and statistical physics, appearing in various forms, essentially equivalent. However, while coarse graining is widely used in astrophysical hydrodynamics and the effect of changing the coarse-graining length has been discussed on several occasions, the exact renormalization group equation does not seem to have appeared before in the astrophysical literature. 8

\section{Dynamical renormalization group}

Dynamical scaling arguments, customary in the physics of surface growth lead one to the conclusion that, when the initial condition is forgotten, the dynamical variables adopt a scaling form such as, for example, $\delta \varrho_{L}:=\varrho_{L}-\langle\varrho\rangle \sim L^{\alpha} f\left(t / L^{z}\right)$, where $\lim _{x \rightarrow \infty} f(x)=1$ and $\lim _{x \rightarrow 0} f(x) \sim x^{\alpha / z}$. In words, the fluctuations grow with time as a power law and they eventually reach saturation, in which state they depend on the coarse-graining length as another power law. An elementary example is the (linear) diffusion equation, in which $z=2$.

The problem is to derive the exponents $\alpha$ and $z$ and the scaling function $f$. Here is where the dynamical RG comes into play: similarly to the situation in static critical phenomena, in which the possible types of critical behaviour correspond to fixed points of the ordinary RG, the possible types of critical dynamics correspond to fixed points of the dynamical RG.

This philosophy is appropriate for the problem on hand, as attested by computer simulations of the microscopic Newtonian mechanics of particles. Indeed, one observes that the fluctuations grow with time. Moreover, if for a fixed time one reduces the coarse-graining length, one observes a similar growth of fluctuations. It is natural to expect that the asymptotic state corresponds to a fixed point of the dynamical RG. Relying on this philosophy, this RG has been employed to determine the fixed points and critical exponents of a simplified hydrodynamic self-gravitating model. 6

Unfortunately, the usual treatment of the dynamical RG is perturbative. This may render it inapplicable for self-gravitating models, as I will argue later. Besides, the fixed point structure depends on the properties of the noise. Both problems

\footnotetext{
${ }^{b}$ Nevertheless, it has been noticed that the point probability distribution $p_{L}(\varrho):=\mathcal{P}_{L}[\varrho(\boldsymbol{r})]$ satip fies a diffusion equation, analogous to Eq. (5), but with only the last term of the right hand side.4 It was used to find the distribution of collapsed objects with volume $L^{3}$ according to their mass.
} 
are overcome by the exact renormalization group as we have derived it: it is nonperturbative and independent of the noise. However, it may depend on the window function. We shall see that this dependence is equivalent to a dependence on the noise and enlightens the role of the latter in the dynamics.

\section{Burgers and Kardar-Parisi-Zhang equations}

In cosmology, it is customary to resort to the Zel'dovich (or parallelism) approximation, which constrains the velocity to haye the same direction that the gravitational field has, that is, $\boldsymbol{v}(\boldsymbol{r}, t)=F(t) \boldsymbol{g}(\boldsymbol{r}, t)$. This approximation greatly simplifies the set of hydrodynamic equations, in fact, reducing them to a single equation for the (redefined) velocity. Furthermore, in the case that the polytropic index $\gamma=2$, this equation becomes the three-dimensional Burgers equation, giving rise to the adhesion model. If If we write the Burgers equation in terms of the velocity (and gravity) potential and we add a noise term, we obtain the Kardar-Parisi-Zhang equation, originally proposed for the description of surface growth. Its fixed point structure corresponding to white noise, as given by the dynamical RG, is wellknown. 5 An even richer structure is obtained by using power-law correlated noise 0

\subsection{Fokker-Planck equation for the Kardar-Parisi-Zhang equation.}

The KPZ equation in Fourier space reads

$$
\frac{d \phi_{\mathbf{k}}}{d t}=F_{\mathbf{k}}\left(\phi_{\mathbf{q}}\right)+\eta_{\mathbf{k}}=-\nu k^{2} \phi_{\mathbf{k}}+\frac{\lambda}{2} \sum_{\mathbf{q}} \boldsymbol{k} \cdot(\boldsymbol{k}-\boldsymbol{q}) \phi_{\mathbf{q}} \phi_{\mathbf{k}-\mathbf{q}}+\eta_{\mathbf{k}} .
$$

It is just a set of coupled Langevin Eqs. If the "force" $F$ were linear, then the equations would decouple and actually reduce to copies of the Langevin Eq. for a Brownian oscillator, which is trivial to solve.

Let us assume Gaussian correlated noise with $\left\langle\eta_{\mathbf{k}}(t) \eta_{\mathbf{k}^{\prime}}\left(t^{\prime}\right)\right\rangle=D(k) \delta_{\mathbf{k},-\mathbf{k}^{\prime}} \delta(t-$ $\left.t^{\prime}\right)$. The Fokker-Planck equation for the probability distribution $\Pi\left(\phi_{\mathbf{k}}, t\right)$ associated to Eqs. (6) is

$$
\frac{\partial \Pi}{\partial t}=-\frac{\partial}{\partial \phi_{\mathbf{k}}}\left[F_{\mathbf{k}} \Pi\right]+\frac{1}{2} D(k) \frac{\partial^{2} \Pi}{\partial \phi_{\mathbf{k}} \partial \phi_{-\mathbf{k}}} .
$$

We can compare it with the exact renormalization group equation, under the assumption of dynamical scaling and, hence, of equivalence of time and scale evolution, that is, $t \leftrightarrow \ln L$ and $\Pi\left(\phi_{\mathbf{k}}, t\right) \leftrightarrow \mathcal{P}_{L}[\varrho]$. We see that both equations are approximately equivalent, if $D(k) \leftrightarrow L\left(d P_{L} / d L\right)$ and $F_{\mathbf{k}}\left(\phi_{\mathbf{q}}\right)$ is linear. The former condition is interesting, for it relates the freedom in the choice of noise with the freedom in the choice of window function, relating the fluctuations with the coarse-graining operation. The latter condition shows that the nonlinearity tends to suppress an exact equivalence.

${ }^{c}$ The one-dimensional Burgers equation had been introduced earlier as a toy model for turbulence. Its 3D counterpart represents pressure-less compressible turbulence without vorticity. 


\section{Critical phenomena and fractals}

The traditional theory of critical phenomena, including critical dynamics, applies to situations close to thermodynamical equilibrium, in which the fluctuations are small. In contrast, here we are dealing with far-from-equilibrium processes that lead to very large fluctuations. In fact, the asymptotic state is probably of fractal nature. In cosmology, a fractal structure of galaxies is currently discernible on small scales. The scale invariance of fractal structures has led to assimilate fractal fluctuations with critical fluctuations and, therefore, the scale at which they subside (homogeneity scale) with the correlation length. This is wrong.

Actually, one can obtain for a particle distribution with two-point correlation $\left(r_{0} / r\right)^{-2 \alpha}$ that the relative fluctuation of the coarse-grained density is given by

$$
\frac{\left\langle\left(\delta \rho_{L}\right)^{2}\right\rangle}{\bar{\rho}^{2}}=\frac{a^{3}}{L^{3}}+B\left(\frac{r_{0}}{L}\right)^{-2 \alpha},
$$

where $a^{3}$ is the volume per particle, and $B$ a number. We clearly see that, for the fractal state, $L \ll r_{0} \Rightarrow \delta \varrho_{L} \gg \bar{\varrho}$, so that thermodynamics is not applicable. Only when $L \gg r_{0}$ thermodynamics becomes applicable as the distribution becomes critical.

When the fluctuations are very large and actually drive the dynamics, the use of perturbation theory is questionable. In this sense, it is also questionable the application of the perturbative dynamical RG to the gravitational interaction. One should instead employ a non-perturbative method, as the exact renormalization group, advocated here.t]

\section{Acknowledgments}

I am grateful to A. Domínguez, J. Pérez-Mercader and L. Pietronero for conversations and to D. Hochberg for a critical reading of this paper.

\section{Appendix: Proof of the exact renormalization group equation}

There are several proofs of the exact renormalization group equation. Here, we adapt the proof provided by T. Morris 10 to our context.

Let us consider the generator of correlations (characteristic function)

$$
Z[J]:=\int \mathcal{D} \varrho \mathcal{P}[\varrho] \exp (-J \cdot \varrho)=\int \mathcal{D} \varrho \exp \left\{-\frac{1}{2} \frac{\varrho_{L}-\underline{\varrho}}{W_{L}} \cdot G^{-1} \cdot \frac{\varrho_{L}-\bar{\varrho}}{W_{L}}-V\left[\varrho_{L}\right]-J \cdot \varrho\right\} .
$$

As we increase $L \rightarrow L+d L$ the dynamical variable $\varrho_{L} \rightarrow \varrho_{L+d L}$, losing detail; that is, losing high wavenumber modes in the Fourier space. Let us define the density $\varrho_{d L}:=\varrho_{L+d L}-\varrho_{L}$, corresponding to the removed high wavenumber modes. In the Fourier space, the filtering convolution becomes a multiplication: $\varrho_{L}(\boldsymbol{k})=W_{L}(\boldsymbol{k}) \varrho(\boldsymbol{k})$. Furthermore, we define the filtered power spectrum 1 $P_{L}(\boldsymbol{k})=G(\boldsymbol{k}) W_{L}(\boldsymbol{k})^{2}$. Hence, $P_{L+d L}=P_{L}+P_{d L}$.

${ }^{d}$ In the more generg field of dynamical critical phenomena, other non-perturbative approaches have been proposed. 
We decompose the integral for the generator at the scale $L+d L$ into two integrals:

$$
Z[J]=\int \mathcal{D} \varrho_{L} \mathcal{D} \varrho_{d L} \exp \left\{-\sum_{\mathbf{k}}\left[\frac{\varrho_{L}^{2}}{2 P_{L}}+\frac{\varrho_{d L}^{2}}{2 P_{d L}}\right]-V\left[\varrho_{L}+\varrho_{d L}\right]-J \cdot\left(\varrho_{L}+\varrho_{d L}\right)\right\} .
$$

Integrating over $\varrho_{d L}$ to get the generator of correlations of $\varrho_{L}$,

$$
\begin{aligned}
Z_{L}\left[\varrho_{L}, J\right]=\int \mathcal{D} \varrho_{d L} & \exp \left\{-\frac{\varrho_{d L}^{2}}{2 P_{d L}}-V\left[\varrho_{L}+\varrho_{d L}\right]-J \cdot\left(\varrho_{L}+\varrho_{d L}\right)\right\} \\
& =\exp \left\{\frac{1}{2} P_{d L} J^{2}-V_{L}\left[-P_{d L} J+\varrho_{L}\right]-J \cdot \varrho_{L}\right\}
\end{aligned}
$$

for a new potential $V_{L}$. (To prove it, use the change of variable $\varrho_{d L}=\varrho_{d L+L}-\varrho_{L}$.) To unravel the meaning of $V_{L}$, take $J(\boldsymbol{k})=0$ for the modes to be removed $(k>$

$1 / L)$. Then $Z_{L}\left[\varrho_{L}, J\right]=\exp \left\{-V_{L}\left[\varrho_{L}\right]-J \cdot \varrho_{L}\right\}$, showing that it is an effective potential.

¿From its definition,

$$
\frac{d}{d L} Z_{L}\left[\varrho_{L}, J\right]=-\frac{1}{2} \frac{d P_{d L}^{-1}}{d L}\left[\frac{\partial}{\partial J(\boldsymbol{k})}+\varrho_{L}\right]^{2} Z_{L}
$$

Substituting for $Z_{L}$ and operating,

$$
\frac{d V_{L}}{d L}=\frac{1}{2} \frac{d P_{L}}{d L}\left[\left(\frac{\partial V_{L}}{\partial \varrho_{L}}\right)^{2}-\frac{\partial^{2} V_{L}}{\partial \varrho_{L}^{2}}\right]=\frac{1}{2} \frac{d P_{L}}{d L} e^{V_{L}} \frac{\partial^{2}}{\partial \varrho_{L}^{2}} e^{-V_{L}}
$$

The first equation is the usual form of the exact renormalization group equation, but the second equality allows us to write it as

$$
\frac{d}{d L} e^{-V_{L}}=-\frac{1}{2} \frac{d P_{L}}{d L} \frac{\partial^{2}}{\partial \varrho_{L}^{2}} e^{-V_{L}}
$$

Adding the quadratic part to get the full $\mathcal{P}$ and substituting for the power spectrum in terms of $W$, we obtain Eq. (5).

\section{References}

1. P.J.E. Peebles, The large scale structure of the universe, Princeton U.P. (1980)

2. A. Domínguez, Phys. Rev. D 62, 103501 (2000)

3. K.G. Wilson and J. Kogut, Phys. Rep. 12, 75 (1974)

4. J.R. Bond, S. Cole, G. Efstathiou and N. Kaiser, Astrophys. J. 379, 440 (1991)

5. A.-L. Barabàsi and H.E. Stanley, Fractal concepts in surface growth, Cambridge U.P. (1995)

6. A. Domínguez, D. Hochberg, J.M. Martín-García, J. Pérez-Mercader and L.S. Schulman, Astron. Astrophys. 344, 27 (1999)

7. J.M. Burgers, The nonlinear diffusion equation, D. Reidel, Dordrecht (1974)

8. J. Gaite, A. Domínguez and J. Pérez-Mercader, Astrophys. J. 522, L5 (1999)

9. C. Castellano, M. Marsili and L. Pietronero, Phys. Rev. Lett. 80, 4830 (1998)

10. T. Morris, Int. Journ. Mod. Phys. A 9, 2411 (1994) 